\title{
Thermal Conductivity of Partially Graphitized Biocarbon Obtained by Carbonization of Medium-Density Fiberboard in the Presence of a Ni-Based Catalyst
}

\author{
T. S. Orlova ${ }^{a, b, *}$, L. S. Parfen'eva ${ }^{a}$, B. I. Smirnov ${ }^{a, * *}$, \\ A. Gutierrez-Pardo ${ }^{c}$, and J. Ramirez-Rico ${ }^{c}$ \\ ${ }^{a}$ Ioffe Physical-Technical Institute, Russian Academy of Sciences, \\ Politeknicheskaya ul. 26, St. Petersburg, 194021 Russia \\ ${ }^{b}$ National Research University of Information Technologies, Mechanics, and Optics, \\ Kronverksky pr. 49, St. Petersburg, 197101 Russia \\ ${ }^{c}$ Departamento de Fisica de la Materia Condensada, Instituto de Ciencia de Materiales de Sevilla (ICMS), \\ Universidad de Sevilla, Sevilla, 41012 Spain \\ *e-mail: orlova.t@mail.ioffe.ru \\ **e-mail: smir.bi@mail.ioffe.ru \\ Received June 17, 2015
}

\begin{abstract}
The thermal conductivity $k$ and resistivity $\rho$ of biocarbon matrices, prepared by carbonizing medium-density fiberboard at $T_{\text {carb }}=850$ and $1500^{\circ} \mathrm{C}$ in the presence of a Ni-based catalyst (samples $\mathrm{MDF}$ $\mathrm{C}(\mathrm{Ni})$ ) and without a catalyst (samples MDF-C), have been measured for the first time in the temperature range of 5-300 K. X-ray diffraction analysis has revealed that the bulk graphite phase arises only at $T_{\text {carb }}=$ $1500^{\circ} \mathrm{C}$. It has been shown that the temperature dependences of the thermal conductivity of samples MDFC-850 and MDF-C-850(Ni) in the range of 80-300 K are to each other and follow the law of $k(T) \sim T^{1.65}$, but the use of the $\mathrm{Ni}$-catalyst leads to an increase in the thermal conductivity by a factor of approximately 1.5 , due to the formation of a greater fraction of the nanocrystalline phase in the presence of the Ni-catalyst at $T_{\text {carb }}=850^{\circ} \mathrm{C}$. In biocarbon MDF-C-1500 prepared without a catalyst, the dependence is $k(T) \sim T^{1.65}$, and it is controlled by the nanocrystalline phase. In MDF-C-1500(Ni), the bulk graphite phase formed increases the thermal conductivity by a factor of 1.5-2 compared to the thermal conductivity of MDF-C-1500 in the entire temperature range of $5-300 \mathrm{~K} ; k(T=300 \mathrm{~K})$ reaches the values of $\sim 10 \mathrm{~W} \mathrm{~m}^{-1} \mathrm{~K}^{-1}$, characteristic of biocarbon obtained without a catalyst only at high temperatures of $T_{\text {carb }}=2400^{\circ} \mathrm{C}$. It has been shown that MDF-C-1500(Ni) in the temperature range of 40-300 K is characterized by the dependence, $k(T) \sim T^{1.3}$, which can be described in terms of the model of partially graphitized biocarbon as a composite of an amorphous matrix with spherical inclusions of the graphite phase.
\end{abstract}

DOI: $10.1134 / \mathrm{S} 1063783416010236$

\section{INTRODUCTION}

Highly porous biomorphic carbon (bio-C) obtained by carbonization (pyrolysis) of natural wood of various origins has caused in recent years a considerable interest due to its favorable combination of natural open porosity and physicomechanical properties of biocarbon materials [1-3]. Depending on the type of the precursor, the total porosity of biomorphic carbon varies from 40 to $75 \%$, with the main pores elongated in the direction of growth of the tree; as for the cross section profile, the pores can be divided into small (with a diameter of up to $10 \mu \mathrm{m}$ ) and large (up to $200 \mu \mathrm{m}$ ). The size and distribution of pores in biomorphic carbon are strongly dependent on the type of precursor (wood). Highly porous biomorphic carbon is used as templates to create new bio- $\mathrm{C}-$ metal or bio$\mathrm{C}$-polymer composites and for the production of light porous biomorphic $\mathrm{SiC}$ ceramics, as well as $\mathrm{SiC}-\mathrm{Si}$ and $\mathrm{SiC}-$ metal composites [4-6]. Biomorphic carbon is considered as a promising material for use as electrodes because of its high porosity, chemical resistance to a variety of electrolytes, and a wide range of operating temperatures. Graphitized carbon matrices draw special attention owing to their thermal properties. Such matrices, along with lightness, have a low coefficient of thermal expansion and high thermal conductivity and are therefore considered as an ideal material for various applications in the field of heat transfer $[7,8]$ and for creating new composite materials with desired properties. Commercial porous graphitized matrices are typically obtained from petroleum 
products. These hydrocarbons can be graphitized at temperatures from 1700 to $3000^{\circ} \mathrm{C}[7,9]$. However, biocarbon materials prepared by conventional carbonization of natural wood are incapable of graphitizing even at a carbonization temperature close to $3000^{\circ} \mathrm{C}$; therefore, they are so-called nongraphitizing carbons $[10,11]$. On the basis of X-ray analysis and research of thermal conductivity and electrical conductivity of these biocarbon materials, it was proposed to consider them as natural composites consisting of an amorphous matrix and nanocrystals of two types: graphite and graphene, the size and volume fraction of which are strongly dependent on the carbonization temperature [12-18]. With $T_{\text {carb }}$ increasing from 800 to $2400^{\circ} \mathrm{C}$, the size of nanocrystals increases from 12 to $25 \AA$ for the graphite components and from 24 to $60 \AA$ for the graphene components [15]; the volume fraction of the nanocrystalline phase also increases [18]. The thermal conductivity of biocarbon materials obtained by carbonizing natural wood of different species (eucalyptus, sapele, beech, pine) and mediumdensity fiberboard without a catalyst was investigated in several studies [15-18]. It was shown that both the thermal conductivity and the temperature dependence of the thermal conductivity strongly depends on the temperature of carbonization. The thermal conductivity of biocarbon materials obtained at $T_{\text {carb }}<800^{\circ} \mathrm{C}$ is determined mainly by the amorphous matrix, while the thermal conductivity of the materials carbonized at $T_{\text {carb }} \geqslant 1000^{\circ} \mathrm{C}$ is determined by the nanocrystalline phase, at least, in the temperature range of $50 \mathrm{~K}<T<$ $300 \mathrm{~K}$.

Recent studies have shown that the bulk graphite phase can be introduced into biocarbon materials carbonized from natural wood, if a catalyst based on transition metals ( $\mathrm{Ni}$ or $\mathrm{Fe}$ ) is used in the carbonization process [19-22]. The elastic, microplastic, strength [23, 24], and electrotransport properties [25] of the partially graphitized carbon obtained by carbonizing beech wood in the presence of a Ni-based catalyst have been studied previously.

In the present study, we investigated the temperature dependence of the thermal conductivity of biocarbon materials obtained by carbonizing mediumdensity fiberboard at $T_{\text {carb }}=850$ and $1500^{\circ} \mathrm{C}$ in the presence of a Ni-catalyst, in comparison with their microstructural features.

\section{SAMPLE PREPARATION AND EXPERIMENTAL TECHNIQUE}

We studied biocarbon samples obtained by carbonizing medium density fiberboard (MDF) at $T_{\text {carb }}=850$ and $1500^{\circ} \mathrm{C}$ in the absence and in the presence of a $\mathrm{Ni}$-based catalyst. A saturated solution of nickel nitrate $\left(4 \mathrm{M} \mathrm{Ni}\left(\mathrm{NO}_{3}\right)_{2}: 6 \mathrm{H}_{2} \mathrm{O}\right)$ in isopropanol was used as a catalyst. Carefully predried pieces of the MDF precursor $75 \times 15 \times 15 \mathrm{~mm}$ in size were placed

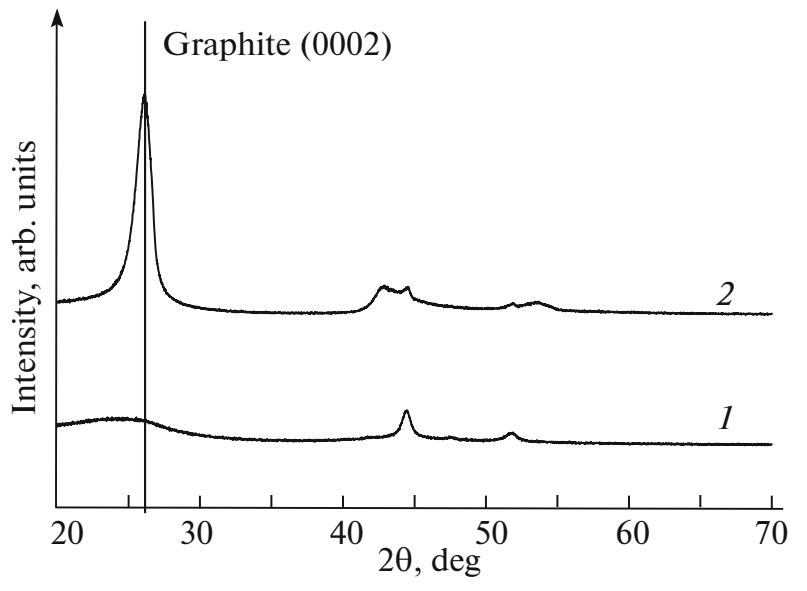

Fig. 1. X-ray diffraction patterns of samples (1) MDF-C$850(\mathrm{Ni})$ and (2) MDF-C-1500(Ni).

in a vacuum chamber and soaked with a solution of the catalyst. Pyrolysis (carbonization) was carried out under nitrogen at a preset temperature of carbonization $T_{\text {carb }}\left(850\right.$ or $\left.1500^{\circ} \mathrm{C}\right)$. After pyrolysis, the particles of Ni-catalyst were removed chemically. The method for obtaining graphitized biocarbon materials is presented in detail in [21]. The biocarbon samples based on the MDF precursor were obtained at the carbonization temperatures of 850 and $1500^{\circ} \mathrm{C}$ with the $\mathrm{Ni}$-catalyst (samples MDF-C-850(Ni) and MDF-C$1500(\mathrm{Ni})$ ) and without a catalyst (samples MDF-C850 and MDF-C-1500).

The structure of the carbonized samples was studied by X-ray diffraction. Figure 1 shows the $\mathrm{X}$-ray diffraction patterns of samples MDF-C-850(Ni) and MDF-C-1500(Ni). It is seen that the use of the Ni-based catalyst resulted in the formation of a graphite phase only in the case of the high carbonization temperature of $T_{\text {carb }}=1500^{\circ} \mathrm{C}$. In the carbonization at $T_{\text {carb }}=850^{\circ} \mathrm{C}$, the samples remained quasi-amorphous. The microstructure of samples derived from MDF with a Ni-based catalyst was studied in detail by scanning and transmission electron microscopy [21]. It has been shown [21] that the graphite phase arises only when the carbonization temperature reaches $\geqslant 1000^{\circ} \mathrm{C}$; the graphite phase is formed as globules, the size and total volume of which increases with increasing carbonization temperature. At $T_{\text {carb }}=1000^{\circ} \mathrm{C}$, the graphite phase is hollow globules $50-100 \mathrm{~nm}$ in size, which are formed by layering graphene around Ni particles and subsequent chemical removal of nickel. When $T_{\text {carb }} \geqslant 1450^{\circ} \mathrm{C}$ (the melting point of nickel), bulk globules of the graphite phase are formed, reaching a size of $0.5-3.0 \mu \mathrm{m}$. The fraction of the resulting graphite phase in the samples obtained at $T_{\text {carb }}=$ $1000^{\circ} \mathrm{C}$ is small, while in MDF-C- $1500(\mathrm{Ni})$, it takes a large part of the sample volume [21]; the proportion of 
the (quasi-)amorphous phase remains large enough (see the data of electron microscopy [21]).

The thermal conductivity $k$ and electrical resistivity $\rho$ were measured in the temperature range of $5-300 \mathrm{~K}$ under vacuum of $10^{-5} \mathrm{~mm} \mathrm{Hg}$ in a setup similar to that used in [26]. Before measurements of the dependences of $k(T)$ and $\rho(T)$, the samples remained in the experiment setup within days under constant pumping to a specified vacuum level to remove moisture and gas that could be present in the pores.

\section{RESULTS AND DISCUSSION}

\subsection{Electrical Resistivity}

The experimental temperature dependences of the electrical resistivity of the MDF biocarbon samples are presented in Fig. 2 disregarding the porosity of the samples; the samples were carbonized at $850^{\circ} \mathrm{C}$ (Fig. 2a) and $1500^{\circ} \mathrm{C}$ (Fig. 2b) in the presence and in the absence of $\mathrm{Ni}$-catalyst. The porosity was taken into account, according to [27], as

$$
\rho^{0}=\rho^{\exp }(1-P),
$$

where $P$ was the porosity of the samples. The values of resistivity $\rho^{0}$ considering the porosity of the samples at room temperature are given in the table. It is seen that the use of the Ni-catalyst did not change the character of the temperature dependence of the resistivity for samples carbonized at both temperatures $\left(T_{\text {carb }}=850\right.$ and $1500^{\circ} \mathrm{C}$ ); however, the conductivity $\sigma=1 / \rho$ of the samples carbonized using the Ni-catalyst was $1.3-$ 2.0 times higher than that of the samples prepared without a catalyst. Previously it was found for biocarbon materials obtained by carbonizing the wood of beech and pine and medium-density fiberboard without a catalyst that the mechanism of charge transfer depends on the temperature of carbonization [28, 29]. In biocarbon matrices obtained at $T_{\text {carb }}<900^{\circ} \mathrm{C}$, the charge transfer occurred by the variable-range hopping conduction mechanism, while the conductivity of materials with $T_{\text {carb }}>1000^{\circ} \mathrm{C}$ is similar to that of a highly disordered metallic system and is well described in terms of the theory of quantum corrections to the conductivity. For the samples with a high temperature of $T_{\text {carb }}=2400^{\circ} \mathrm{C}$, in which the formation of twodimensional layers and larger crystallites of graphite is observed, the role of the two-dimensional conductivity increases [28]. Analysis of the temperature dependences of the resistivity of the samples showed that in the case of MDF-C-850(Ni), the conductivity is still well described by the variable-range hopping conduction mechanism (Fig. 3a). The use of the Ni-catalyst did not change the character of dependence $\rho(T)$ in the case of high carbonization temperature of $T_{\text {carb }}=$ $1500^{\circ} \mathrm{C}$ (Fig. 3b), despite the fact that in this case, as shown by the studies of the microstructure, a significant proportion of the bulk graphite phase is introduced [21]. A detailed study of the impact of the intro-
Thermal conductivity $k_{\mathrm{ph}}^{0}$ and resistivity $\rho^{0}$ of biocarbon samples with regard to their porosity

\begin{tabular}{l|c|l|c}
\hline \multicolumn{1}{c|}{ Sample } & $\begin{array}{c}k_{\mathrm{ph}}^{0}, \\
\mathrm{~W} \mathrm{~m}^{-1} \mathrm{~K}^{-1} \\
(300 \mathrm{~K})\end{array}$ & $\begin{array}{c}\rho^{0}, \Omega \mathrm{cm} \\
(300 \mathrm{~K})\end{array}$ & References \\
\hline MDF-C-850 & 2.43 & 0.158 & This work \\
MDF-C-850(Ni) & 3.84 & 0.1317 & The same \\
MDF-C-1500 & 4.45 & 0.017 & $»$ \\
MDF-C-1500(Ni) & 10.37 & 0.0106 & $»$ \\
PI-C-2400 & 12.65 & 0.007 & {$[13]$} \\
BE-C-2400 & 9.65 & 0.0065 & {$[15]$} \\
\hline
\end{tabular}

duced graphite phase on the electrotransport properties of beech wood biocarbon is reported in [25].
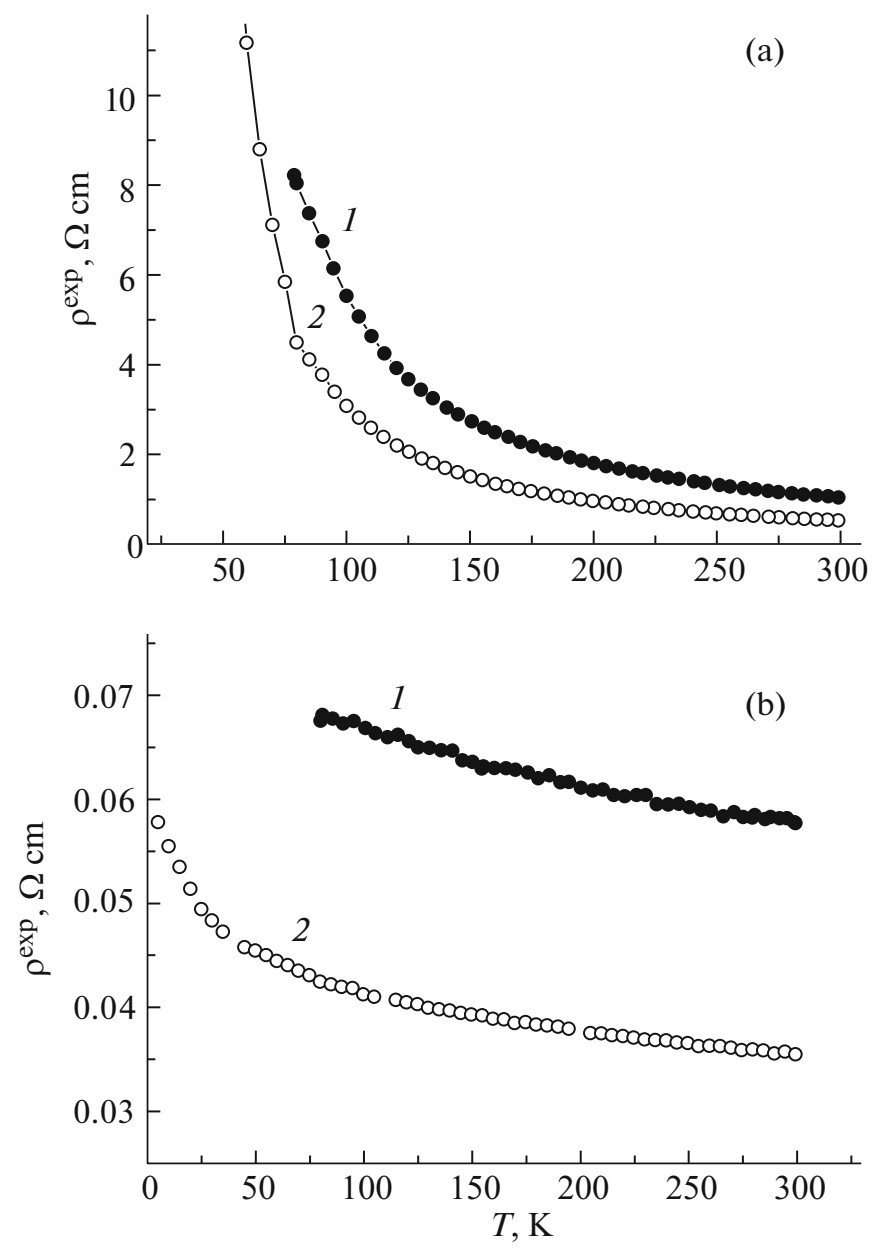

Fig. 2. Temperature dependences of the electrical resistivity $\rho^{\exp }(T)$ of biocarbon samples obtained by carbonization of MDF at (a) 850 and (b) $1500^{\circ} \mathrm{C}$ in the (1) absence and (2) presence of Ni-catalyst; the data are presented disregarding the porosity. 

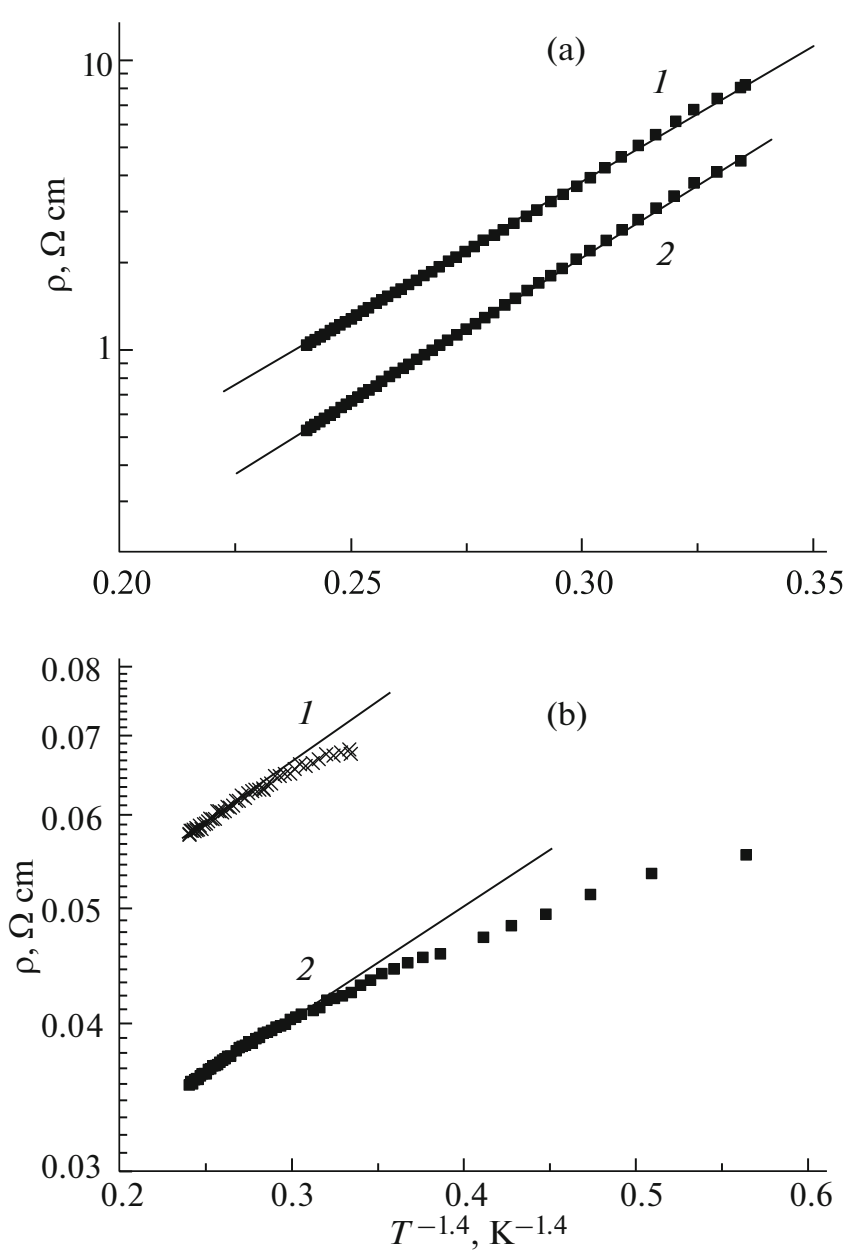

Fig. 3. Temperature dependences of the electrical resistivity of biocarbon samples obtained by carbonization of MDF at (a) 850 and (b) $1500^{\circ} \mathrm{C}$ in the (1) absence and (2) presence of a Ni-catalyst, plotted in the coordinates of $\log \rho^{\exp }-T^{-1.4}$.

\subsection{Thermal Conductivity}

Figure 4 demonstrates the temperature dependences of the total thermal conductivity $k^{\exp }$ of the samples of MDF biocarbon matrices, obtained without a catalyst (curves 1 and 2) and with the Ni-catalyst (curves 3 and 4 ) at $T_{\text {carb }}=850$ and $1500^{\circ} \mathrm{C}$. The results are presented without taking into account the porosity of the samples.

The total thermal conductivity measured in the experiment consists of the electronic $k_{e}$ and phononic $k_{\mathrm{ph}}$ components, that is,

$$
k^{\text {exp }}=k_{e}+k_{\mathrm{ph}} \text {. }
$$

The evaluations of $k_{e}$ by the Wiedemann-Franz law

$$
k_{e}=L T \sigma
$$

( $L$ is the Lorentz number) show that over the entire temperature range $(5-300 \mathrm{~K})$, even with the highest number of $L=L_{0}=2.45 \times 10^{-8} \mathrm{~W} \Omega \mathrm{K}^{-2}$, the value of

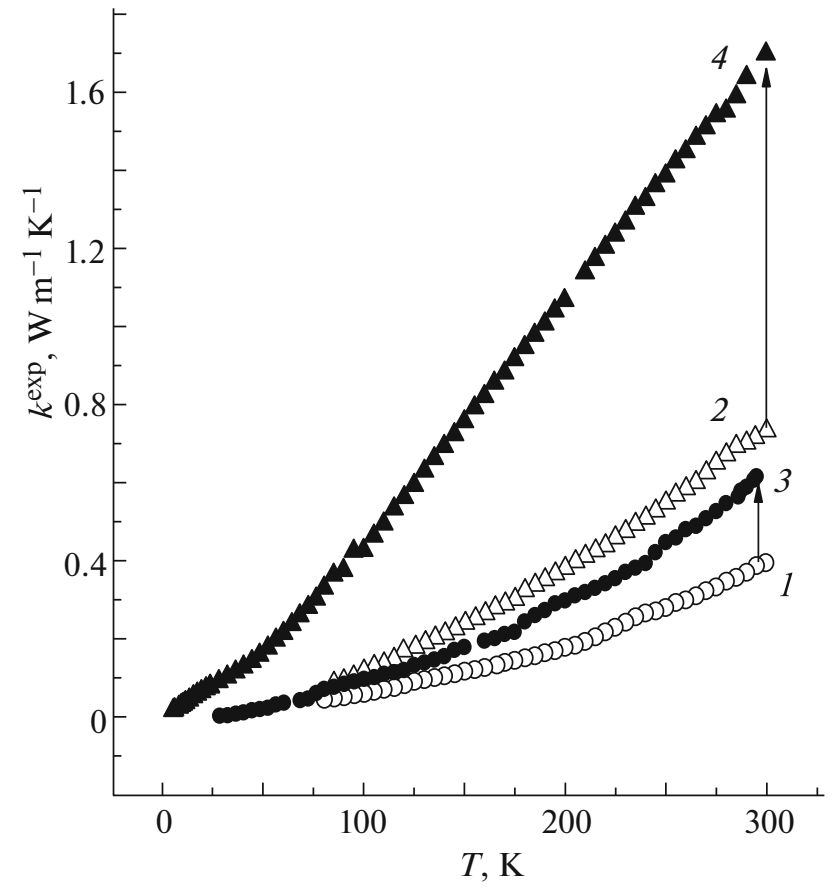

Fig. 4. Temperature dependences of the total thermal conductivity $k^{\mathrm{exp}}$ of the samples of MDF biocarbon matrices, obtained ( 1 and 2 ) without a catalyst and ( 3 and 4 ) with the $\mathrm{Ni}$-catalyst at $T_{\text {carb }}=(1,3) 850$ and $(2,4) 1500^{\circ} \mathrm{C}$; the data are presented disregarding the porosity of the samples; arrows indicate changes in the thermal conductivity at $300 \mathrm{~K}$ because of the use of the Ni-catalyst.

$k_{\mathrm{ph}}$ is more than two orders of magnitude higher than $k_{e}$. Thus, $k^{\exp } \approx k_{\mathrm{ph}}$.

To analyze the thermal conductivity of the biocarbon materials themselves (biocarbon skeletons), we considered the porosity of the samples in the same way as it was done in a number of previous studies [15-18], using equation [30]

$$
k^{\exp } \approx k_{\mathrm{ph}}=k_{\mathrm{ph}}^{0}(1-P) \sqrt{1-P},
$$

where $k^{\mathrm{exp}}$ and $k_{\mathrm{ph}}^{0}$ are the thermal conductivities without and with taking into account the sample porosity $P$, respectively. The temperature dependences of thermal conductivity $k_{\mathrm{ph}}^{0}(T)$, taking into account the porosity of MDF-C and MDF-C(Ni), obtained at the carbonization temperatures of 850 and $1500^{\circ} \mathrm{C}$, are given in the logarithmic scale in Figs. 5 and 6, respectively. The temperature dependences of the thermal conductivity of MDF-C-850 and MDF-C-850(Ni) in the range of $80-300 \mathrm{~K}$ are similar and follow the law of $k(T) \sim T^{1.65}$. According to [18], this means that in this temperature range, the nanocrystalline phase is responsible for heat transfer (dissipation). The use of the Ni-catalyst at $T_{\text {carb }}=850^{\circ} \mathrm{C}$ did not lead to the introduction of the bulk graphite phase (Fig. 1), but appears to increase the total volume or size of nanocrystals, which increased the thermal conductivity in 


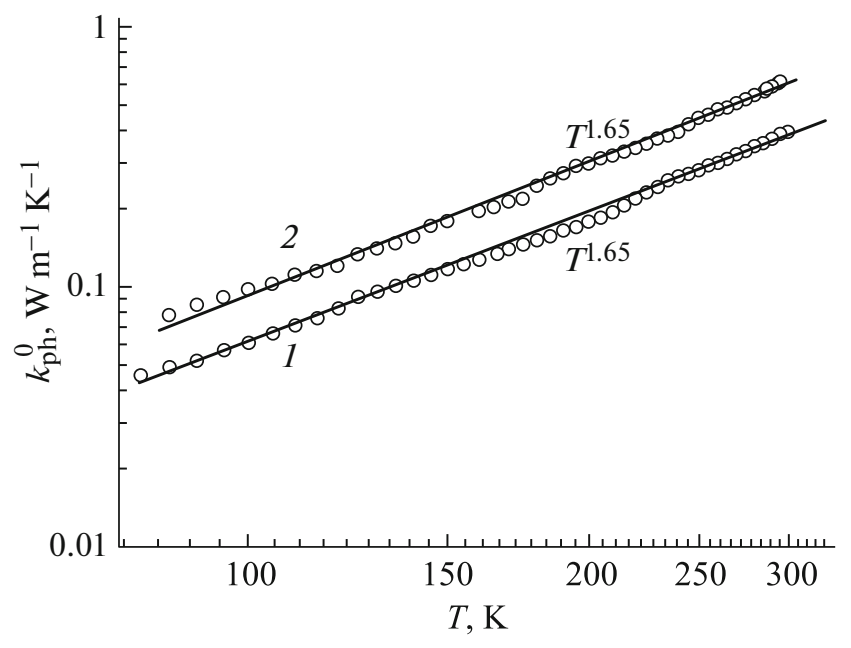

Fig. 5. Temperature dependences of the thermal conductivity $k_{\mathrm{ph}}^{0}$ for the samples of MDF biocarbon matrices, obtained in the (1) absence and (2) presence of Ni-catalyst at $T_{\text {carb }}=850^{\circ} \mathrm{C}$; the data are presented taking into account the porosity of the samples on a logarithmic scale.

the temperature range of $80-300 \mathrm{~K}$ by approximately 1.5 times.

The temperature dependence of the thermal conductivity of MDF-C-1500 also follows the law of $k(T) \sim T^{1.65}$ in the range of 80-300 K (Fig. 6, curve 1) and is determined by the nanocrystalline phase. Such dependences of $k(T) \sim T^{n}(n=1.6 \pm 0.1)$ were observed previously in biocarbon materials obtained by carbonizing wood of beech (BE-C-2400) [15] (Fig. 6, curve 3) and pine (PI-C-2400) [13] at $T_{\text {carb }}=$ $2400^{\circ} \mathrm{C}$ without a catalyst. The experimentally observed dependence of $k(T) \sim T^{1.65}$ for biocarbon MDF-C-1500 is close to the dependence of $T^{2}$, characteristic of $2 \mathrm{D}$-disordered carbon nanostructures [31]. The deviation of value $n=1.6 \pm 0.1$ from $n=2.0$ may be because in biomorphic carbon, even at high carbonization temperature $\left(2400^{\circ} \mathrm{C}\right)$, the size of nanocrystals remains ultrasmall: $D \approx 60 \AA[13,15]$. Then, according to [31], the heat transfer is controlled by the internal properties of intercrystalline boundaries.

Heat is usually transferred in carbon mainly by acoustic phonons. In disordered and nanostructured materials, the thermal properties, namely, thermal conductivity $k$, are limited by the disordering and intercrystalline boundaries rather than the intrinsic properties inherent in the lattice (lattice dynamics) [31]. This behavior of thermal conductivity is characteristic, for example, of ultrananocrystalline diamond [31]. Diamond-like carbon (DLC) is a metastable form of amorphous carbon containing a substantial portion of $s p^{3}$-bonds. DLC with the highest concentration of $s p^{3}$-bonds is called tetrahedral amorphous carbon. Tetrahedral amorphous carbon refers to mate-

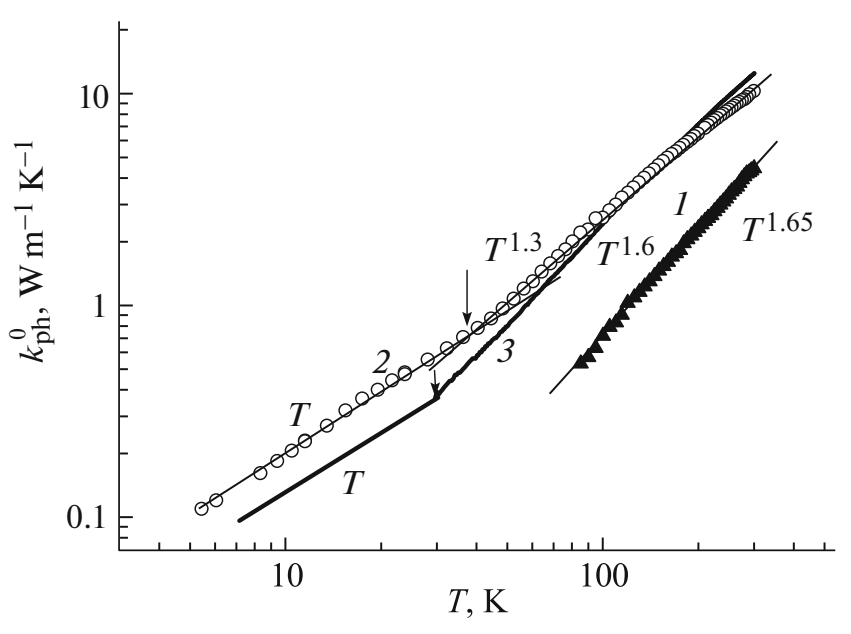

Fig. 6. Temperature dependences of the thermal conductivity $k_{\mathrm{ph}}^{0}$ for the samples of MDF biocarbon matrices, obtained in the (1) absence and (2) presence of Ni-catalyst at $T_{\text {carb }}=1500^{\circ} \mathrm{C}$; (3) the data [15] for BE-C-2400 biocarbon obtained by carbonization of beech wood at $T_{\text {carb }}=$ $2400^{\circ} \mathrm{C}$ without a catalyst; the data are presented taking into account the porosity of the samples on a logarithmic scale.

rials having the greatest $k$ value among amorphous solids, reaching $\sim 10 \mathrm{~W} \mathrm{~m}^{-1} \mathrm{~K}^{-1}$ at room temperature [31]. The experimentally obtained values of $k \approx$ $4.6 \mathrm{~W} \mathrm{~m}^{-1} \mathrm{~K}^{-1}$ for MDF-C-1500 (this work), $k \approx 12.6 \mathrm{~W} \mathrm{~m}^{-1} \mathrm{~K}^{-1}$ for PI-C-2400 [13], and $k \approx$ $9.7 \mathrm{~W} \mathrm{~m}^{-1} \mathrm{~K}^{-1}$ for BE-C-2400 [15] at room temperature are comparable with a thermal conductivity of tetrahedral amorphous carbon, while in the conventional amorphous carbon, $k$ increases from $\sim 0.01 \mathrm{~W} \mathrm{~m}^{-1} \mathrm{~K}^{-1}$ at $T=4 \mathrm{~K}$ only to $\sim 2 \mathrm{~W} \mathrm{~m}^{-1} \mathrm{~K}^{-1}$ at $T=500 \mathrm{~K}$. Thus, although the biomorphic carbon materials obtained by carbonizing natural wood at high temperatures without a catalyst are X-ray amorphous carbon, they are close to ultrananocrystalline diamond or diamondlike carbon by the character of the temperature dependence and the thermal conductivity at room temperature.

In samples MDF-C-1500(Ni), a real bulk graphite phase arises (Fig. 1). It is seen from the electron microscopic images [21] that the graphite phase consists of globules with sizes from submicron and up to $\sim 1-2 \mu \mathrm{m}$, and its volume fraction is significantly less than half of the sample volume. The temperature dependence of the thermal conductivity of MDF-C$1500(\mathrm{Ni})$ (Fig. 6, curve 2) is described by the relation of $k \sim T$ in the temperature range of $T<40 \mathrm{~K}$ and $k \sim$ $T^{1.3}$ in the range of $40 \mathrm{~K}<T<300 \mathrm{~K}$.

The dependence of $k \sim T^{1.3}$ for MDF-C-1500(Ni) is closer to the dependence of $k \sim T$, characteristic of an amorphous phase, than the dependence of $k \sim T^{n}$ $(n=1.6 \pm 0.1)$, characteristic of MDF-C-1500, BE- 
C-2400 [15], and PI-C-2400 [13]. The resulting dependence of $k \sim T^{1.3}$ supports our assumption made in the study of elastic [23] and strength properties [24] of partially graphitized beech wood carbon that the emerging phase of bulk graphite inhibits to some extent the formation of the nanocrystalline phase, causing the formed graphite globules "float" in an amorphous medium.

To describe the thermal conductivity of the composite structure, consisting of a matrix with spherical inclusions of the second phase, a theory of effective medium was developed [32]. According to [32], the thermal conductivity of such a composite can be estimated as

$$
\begin{gathered}
4 k=\left(3 f_{2}-1\right) k_{2}+\left(3 f_{1}-1\right) k_{1} \\
+\left[\left(\left(3 f_{2}-1\right) k_{2}+\left(3 f_{1}-1\right) k_{1}\right)^{2}+8 k_{1} k_{2}\right]^{1 / 2},
\end{gathered}
$$

where $k_{1}$ is the thermal conductivity of the matrix, $k_{2}$ is the thermal conductivity of the second phase, and $f_{1}$ and $f_{2}$ are the volume fraction of the matrix and the second phase, respectively. The thermal conductivity of partially graphitized carbon MDF-C-1500(Ni) was evaluated in the framework of the model [32], based on the following parameters: $k_{G}=500-1500 \mathrm{~W} \mathrm{~m}^{-1} \mathrm{~K}^{-1}$ were taken as the thermal conductivity of graphite inclusions, typical of synthesized graphite [33, 34], and $k_{\mathrm{am}} \sim 0.3 \mathrm{~W} \mathrm{~m}^{-1} \mathrm{~K}^{-1}[31,35]$ was the thermal conductivity of amorphous carbon. The good agreement between the experimentally measured value of $k^{\exp } \approx$ $k_{\mathrm{ph}}^{0} \approx 10 \mathrm{~W} \mathrm{~m}^{-1} \mathrm{~K}^{-1}$ and the resulting theoretical estimate of $k^{\text {th }}=8-17 \mathrm{~W} \mathrm{~m}^{-1} \mathrm{~K}^{-1}$ is reached for the volume fraction of the graphite phase of $f_{2} \sim 0.33-0.35$. Although in this work, the exact value of $f_{2}$ cannot be measured, it is seen from the electron microscopic images of the structure of the test samples (MDF-C$1600(\mathrm{Ni}))$ [21] that the values of $f_{2} \sim 0.33-0.35$ are in good enough accordance with the actual fraction of the graphite phase. It is noteworthy that the thermal conductivity of pine wood [13] and beech biocarbon [15], carbonized at $2400^{\circ} \mathrm{C}$, are comparable (or even approximately $20 \%$ higher in the case pine wood biocarbon) with $k$ for MDF-C-1500(Ni) (table), despite the fact that in the former case, the samples remain $\mathrm{X}$ ray amorphous, whereas in MDF-C-1500(Ni), a bulk graphite phase is formed (Fig. 1). Although the presence of the graphite phase significantly (by $\sim 1.5$-fold) increases the thermal conductivity of MDF-C$1500(\mathrm{Ni})$ as compared with the value typical of MDF$\mathrm{C}-1500$, the graphite phase is not dominant in these samples and does not introduce, apparently, a defining contribution to the temperature dependence of thermal conductivity. The question of whether the introduction of a significant portion or fraction of the graphite phase in the biocarbon with increasing carbonization temperature in the presence of $\mathrm{Ni}$-catalyst or by using some other more effective catalyst remains open and requires further research.

\section{CONCLUSIONS}

On the basis of these structural studies and measurements in the temperature range of 5-300 K of the electrical conductivity and thermal conductivity of highly porous ( $\sim 65$ vol $\%)$, partially graphitized biocarbon matrices, prepared by carbonizing mediumdensity fiberboard at $T_{\text {carb }}=850$ and $1500^{\circ} \mathrm{C}$ in the presence of a Ni-based catalyst (samples MDF$\mathrm{C}(\mathrm{Ni})$ ) and without a catalyst (samples MDF-C), the following conclusions can be drawn.

(1) X-ray diffraction analysis showed that the bulk graphite phase arises only at $T_{\text {carb }}=1500^{\circ} \mathrm{C}$; the use of a Ni-catalyst at $T_{\text {carb }}=850^{\circ} \mathrm{C}$ is ineffective.

(2) The temperature dependences of the resistivity of the samples of MDF-C(Ni) biocarbon matrices, carbonized at $T_{\text {carb }}=850$ and $1500^{\circ} \mathrm{C}$ in the presence of Ni-based catalyst, are studied in the temperature range of 5-300 K. It is shown that in both cases, the use of Ni-catalyst did not change the semiconductor nature of dependencies $\rho(T)$, but the conductivity increased 1.3-2.0 times. The temperature dependence of the resistivity of MDF-C-850(Ni) is well described by variable-range hopping conduction mechanism.

(3) The thermal conductivity of MDF-C and MDF-C(Ni), carbonized at $T_{\text {carb }}=850$ and $1500^{\circ} \mathrm{C}$ is studied in the temperature range of $5-300 \mathrm{~K}$. The temperature dependences of the thermal conductivity of samples MDF-C-850, and MDF-C-850(Ni), and MDF-C-1500 in the range of $80 \mathrm{~K}<T_{\text {carb }}<300 \mathrm{~K}$ follow the law of $k(T) \sim T^{1.65}$, characteristic of the nanocrystalline phase [18]. In samples MDF-C-1500(Ni), the thermal conductivity increases with temperature linearly $(k(T) \sim T)$ in the range of $T<40 \mathrm{~K}$ and then it follows the law of $k(T) \sim T^{1.3}$ at $T>40 \mathrm{~K}$. This behavior of thermal conductivity is most likely characteristic of the composite structure consisting of an amorphous matrix with inclusions of graphite globules. The evaluation of the thermal conductivity of samples MDF$\mathrm{C}-1500$ (Ni) at room temperature under the effective medium theory developed to describe the thermal conductivity of the composite structure, which is a matrix with spherical inclusions of the second phase, gives a good agreement with experimental results. These results support the hypothesis that the emerging bulk graphite phase leads to the suppression of the formation of the nanocrystalline phase.

\section{ACKNOWLEDGMENTS}

This work was initiated by I.A. Smirnov. The authors are grateful for $\mathrm{H}$. Misiorek for his help in the measurement of thermal conductivity. 
This study was supported in part by the Russian Foundation for Basic Research (project no. 14-0300496).

\section{REFERENCES}

1. P. Greil, T. Lifka, and A. Kaindl, J. Eur. Ceram. Soc. 18 (14), 1961 (1998).

2. P. Greil, J. Eur. Ceram. Soc. 21 (2), 105 (2001).

3. V. S. Kaul, K. T. Faber, R. Sepulveda, A. R. de Arellano Lopez, and J. Martinez-Fernandez, Mater. Sci. Eng., A 428 (1-2), 225 (2006).

4. A. R. de Arellano-Lopez, J. Martinez-Fernandez, P. Gonzalez, C. Dominguez, V. Fernandez-Quero, and M. Singh, Int. J. Appl. Ceram. Technol. 1 (1), 56 (2004).

5. F. M. Varela-Feria, J. Martinez-Fernandez, A. R. de Arellano-Lopez, and M. Singh, J. Eur. Ceram. Soc. 22 (14-15), 2719 (2002).

6. C. E. Byrne and D. C. Nagle, Carbon 35 (2), 259 (1997).

7. J. Klett, R. Hardy, E. Romine, C. Walls, and T. Burchell, Carbon 38 (7), 953 (2000).

8. D. Gaies and K. T. Faber, Carbon 40 (7), 1137 (2002).

9. R. E. Franklin, Acta Crystallogr. 4 (5), 235 (1951).

10. C. E. Byrne and D. C. Nagle, Carbon 35 (2), 267 (1997).

11. H. M. Cheng, H. Endo, T. Okabe, K. Saito, and G. B. Zheng, J. Porous Mater. 6 (3), 233 (1999).

12. L. S. Parfen'eva, T. S. Orlova, N. F. Kartenko, N. V. Sharenkova, B. I. Smirnov, I. A. Smirnov, H. Misiorek, A. Jezowski, J. Mucha, A. R. de ArellanoLopez, J. Martinez-Fernandez, and F. M. VarelaFeria, Phys. Solid State 48 (3), 441 (2006).

13. L. S. Parfen'eva, T. S. Orlova, N. F. Kartenko, N. V. Sharenkova, B. I. Smirnov, I. A. Smirnov, H. Misiorek, A. Jezowski, T. E. Wilkes, and K. T. Faber, Phys. Solid State 50 (12), 2245 (2008).

14. L. S. Parfen'eva, T. S. Orlova, N. F. Kartenko, N. V. Sharenkova, B. I. Smirnov, I. A. Smirnov, H. Misiorek, A. Jezowski, J. Mucha, A. R. de ArellanoLopez, and J. Martinez-Fernandez, Phys. Solid State 51 (10), 2023 (2009).

15. L. S. Parfen'eva, T. S. Orlova, N. F. Kartenko, N. V. Sharenkova, B. I. Smirnov, I. A. Smirnov, H. Misiorek, A. Jezowski, T. E. Wilkes, and K. T. Faber, Phys. Solid State 52 (6), 1115 (2010).

16. L. S. Parfen'eva, T. S. Orlova, N. F. Kartenko, B. I. Smirnov, I. A. Smirnov, H. Misiorek, A. Jezow- ski, J. Muha, and M. C. Vera, Phys. Solid State 53 (11), 2398 (2011).

17. L. S. Parfen'eva, T. S. Orlova, B. I. Smirnov, I. A. Smirnov, H. Misiorek, A. Jezowski, and J. Ramirez-Rico, Phys. Solid State 56 (5), 1071 (2014).

18. N. F. Kartenko, T. S. Orlova, L. S. Parfen'eva, B. I. Smirnov, and I. A. Smirnov, Phys. Solid State 56 (11), 2348 (2014).

19. M. T. Johnson and K. T. Faber, J. Mater. Res. 26 (1), 18 (2011).

20. M. T. Johnson, A. S. Childers, J. Ramirez-Rico, H. Wang, and K. T. Faber, Composites, Part A 53, 182 (2013).

21. A. Gutierrez-Pardo, J. Ramirez-Rico, A. R. de Arellano-Lopez, and J. Martinez-Fernandez, J. Mater. Sci. 49, 22 (2014).

22. A. Gutierrez-Pardo, J. Ramirez-Rico, R. CabezasRodriguez, and J. Martinez-Fernandez, J. Power Sources 278, 18 (2015).

23. T. S. Orlova, B. K. Kardashev, B. I. Smirnov, A. Gutierrez-Pardo, J. Ramirez-Rico, and J. Martinez-Fernandez, Phys. Solid State 57 (3), 586 (2015).

24. V. V. Shpeizman, T. S. Orlova, B. I. Smirnov, A. Gutierrez-Pardo, and J. Ramirez-Rico, Mater. Phys. Mech. 21, 200 (2014).

25. V. V. Popov, T. S. Orlova, A. Gutierrez-Pardo, and J. Ramirez-Rico, Phys. Solid State 57 (9), 1746 (2015).

26. A. Jezowski, J. Mucha, and G. Pompe, J. Phys. D: Appl. Phys. 20, 1500 (1987).

27. A. L. Love, J. Appl. Phys. 22, 252 (1951).

28. V. V. Popov, T. S. Orlova, and J. Ramirez-Rico, Phys. Solid State 51 (11), 2247 (2009).

29. V. V. Popov, T. S. Orlova, E. Enrique Magarino, M. A. Bautista, and J. Martinez-Fernandez, Phys. Solid State 53 (2), 276 (2011).

30. E. Ya. Litovskii, Izv. Akad. Nauk SSSR, Neorg. Mater. 16, 559 (1980).

31. A. A. Balandin, Nat. Mater. 10, 569 (2011).

32. A. J. Bullen, K. E. O'Hara, and D. G. Cahill, J. Appl. Phys. 88, 6317 (2000).

33. D. T. Morelli and G. A. Slack, in High Thermal Conductivity Materials, Ed. by S. L. Shinde and J. S. Goela (Springer-Verlag, New York, 2006), p. 37.

34. N. C. Gallego and J. W. Klett, Carbon 41, 1461 (2003).

35. Handbook of Physical Quantities, Ed. by I. S. Grigoriev and E. Z. Meilikhov (Energoizdat, Moscow, 1991; CRC Press, Boca Raton, Florida, United States, 1996).

Translated by $O$. Zhukova 